\title{
Strategic Distributional Cost-Effectiveness Analysis for Improving National Cancer Screening Uptake in Cervical Cancer: A Focus on Regional Inequality in South Korea
}

\author{
Tae-Hoon Lee, MPH',2 \\ Woorim Kim, BSc, ${ }^{1,2}$ \\ Jaeyong Shin, MD, MPH',3 \\ Eun-Cheol Park, MD, PhD2,3 \\ Sohee Park, PhD2,4 \\ Tae Hyun Kim, $\mathrm{PhD}^{24}$
}

${ }^{1}$ Department of Public Health, Graduate School, Yonsei University, Seoul, ${ }^{2}$ Institute of Health Services Research, Yonsei University, Seoul, ${ }^{3}$ Department of Preventive Medicine, Yonsei University College of Medicine, Seoul, ${ }^{4}$ Graduate School of Public Heath, Yonsei University, Seoul, Korea
Correspondence: Tae Hyun Kim, PhD Graduate School of Public Health, Yonsei University, 50 Yonsei-ro, Seodaemun-gu, Seoul 03722, Korea Tel: 82-2-2228-1521

Fax: 82-2-392-8133

E-mail: THKIM@yuhs.ac

Received November 3, 2016

Accepted March 20, 2017

Published Online March 30, 2017

*Tae-Hoon Lee and Woorim Kim contributed equally to this work.

\section{Purpose}

The purpose of this study was to conduct a cost effectiveness analysis of strategies designed to improve national cervical cancer screening rates, along with a distributional cost effectiveness analysis that considers regional disparities.

\section{Materials and Methods}

Cost effectiveness analysis was conducted using a Markov cohort simulation model, with quality adjusted life years as the unit of effectiveness. The strategies considered were current (biennial Papanicolaou smear cytology of females aged 20 or above), strong screening recommendation by mail to target regions (effect, $12 \%$ increase in screening uptake; cost, 1,000 Korean won per person), regular universal screening recommendation by mail (effect, 6\% increase in screening uptake; cost, 500 Korean won per person), and strong universal screening recommendation by mail (effect, $12 \%$ increase in screening uptake; cost, 1,000 Korean won per person). Distributional cost effectiveness analysis was conducted by calculating the cost effectiveness of strategies using the Atkinson incremental cost effectiveness ratio.

\section{Results}

All strategies were under the threshold value, which was set as the Korean gross domestic product of $\$ 25,990$. In particular, the 'strong screening recommendation to target regions' strategy was found to be the most cost effective (incremental cost effectiveness ratio, $7,361,145$ Korean won). This was also true when societal inequality aversion increased in the distributional cost effectiveness analysis.

\section{Conclusion}

The 'strong screening recommendation to target regions' strategy was the most cost effective approach, even when adjusting for inequality. As efficiency and equity are objectives concurrently sought in healthcare, these findings imply a need to develop appropriate economic evaluation methodologies to assess healthcare policies.

\section{Introduction}

A significant proportion of deaths in South Korea can be attributed to cancer, with cancer mortality rates being reported as 150.9 per 100,000 individuals in 2014 [1]. Cancer is known to impose large socioeconomic burdens on society
Key words

National cervical cancer screening, Cost-benefit analysis,

Distributional cost effectiveness analysis,

Screening uptake regional disparities, Healthcare disparities 


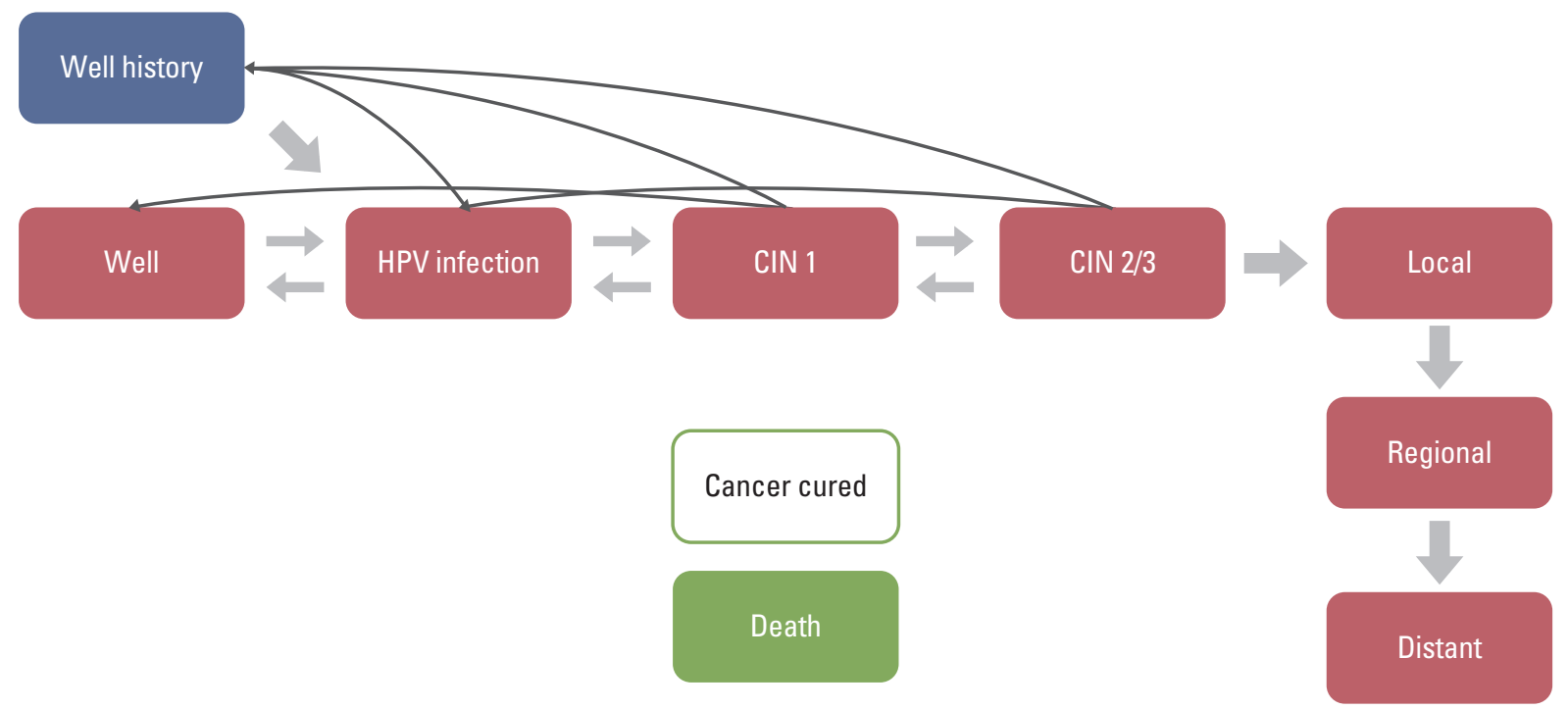

Fig. 1. Model of the natural history of cervical cancer. HPV, human papillomavirus; CIN, cervical intraepithelial neoplasia.

The World Health Organization reported that a 93\% reduction in the incidence of cervical cancer can be expected in response to introduction of screening programs [3]. Moreover, previous studies have shown that national cervical cancer screening is cost effective at reducing cervical cancer incidence and mortality in South Korea [4,5]. Cho et al. [6] demonstrated that the national cervical cancer screening program was effective in terms of life years saved (LYS), with a threshold of $\$ 32,272$ ( $\$ 1=1,100$ Korean won [KRW]) per person, which was the gross domestic product (GDP) of South Korea in 2012 (incremental cost effectiveness ratio [ICER], 7,581,679 KRW/LYS). Similarly, another study cited the annual conduction of Papanicolaou smear cytology (Pap) to females aged 30 or above as the most cost effective strategy in quality adjusted life years (QALY) [7].

Despite the potential benefits of national cervical cancer screening uptake, examination rates are reportedly lower in South Korea (approximately 66\%) than in other countries including the United States (74\%) and the United Kingdom (78\%) [8]. Disparities were also found among individuals of various socioeconomic status, and particularly among those of different regional status [8]. Hence, such tendencies require the need for a cost effectiveness analysis that takes into account regional inequality and distribution of health effects, in addition to previous research that has aimed to maximize population health using restricted resources [9].

Despite such importance, few South Korean studies have included cost effectiveness analysis of cervical cancer based on regional disparities in cancer screening. A previous study targeting Taiwanese participants concluded that individuals who received group education programs had higher screening rates than those who received introduction pamphlets through the mail [10]. Another study in the United States demonstrated that sending introduction pamphlets by mail can increase cervical cancer screening rates by about $12 \%$ [11]. Therefore, this study was conducted to evaluate cost effectiveness of methods with the aim of identifying strategies that can improve the South Korean national cervical cancer screening uptake rate. Further analysis was also performed considering regional disparities in screening rates using the distributional cost effectiveness analysis method, which specifically considers health inequalities under the cost effectiveness framework [12].

\section{Materials and Methods}

\section{Model structure: cost-effectiveness analysis}

A Markov simulation model was developed using TreeAge Pro (TreeAge Software, Williamstown, MA). The model structure is illustrated in Fig. 1. Individuals enter the model in a healthy, well state. With time, a proportion of the individuals are found to be susceptible to human papillomavirus (HPV) infection, which can progress to cervical intraepithelial neoplasia (CIN) and cancer.

The model incorporates disease regression, and individuals immediately diagnosed with CIN move to the well his- 


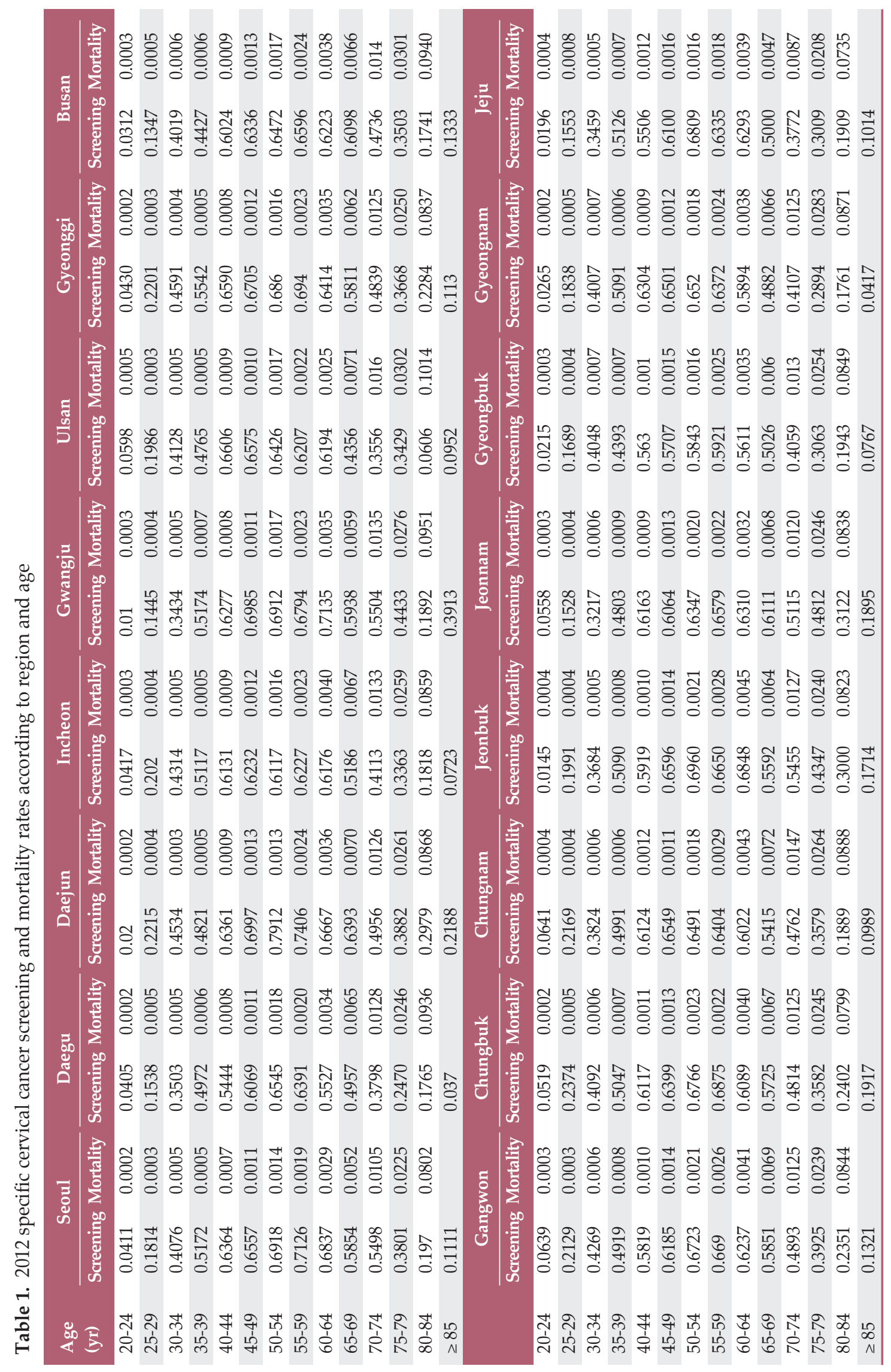


Table 2. Age- and site-specific cervical cancer mortality rates

\begin{tabular}{|c|c|c|c|c|c|}
\hline \multirow{2}{*}{ Age (yr) } & \multicolumn{5}{|c|}{ Mortality rate } \\
\hline & 1-Year & 2-Year & 3-Year & 4-Year & 5-Year \\
\hline \multicolumn{6}{|l|}{ Local } \\
\hline $20-29$ & 0.039 & 0.093 & 0.127 & 0.147 & 0.167 \\
\hline $30-39$ & 0.017 & 0.057 & 0.089 & 0.105 & 0.117 \\
\hline $40-49$ & 0.013 & 0.038 & 0.062 & 0.073 & 0.091 \\
\hline $50-59$ & 0.015 & 0.054 & 0.089 & 0.118 & 0.140 \\
\hline $60-69$ & 0.027 & 0.067 & 0.114 & 0.143 & 0.157 \\
\hline $70-79$ & 0.048 & 0.115 & 0.146 & 0.191 & 0.214 \\
\hline$\geq 80$ & 0.113 & 0.223 & 0.357 & 0.392 & 0.567 \\
\hline \multicolumn{6}{|l|}{ Regional } \\
\hline $20-29$ & 0.177 & 0.155 & 0.057 & 0.000 & 0.035 \\
\hline $30-39$ & 0.100 & 0.131 & 0.055 & 0.043 & 0.026 \\
\hline $40-49$ & 0.087 & 0.133 & 0.072 & 0.035 & 0.024 \\
\hline $50-59$ & 0.090 & 0.103 & 0.073 & 0.034 & 0.033 \\
\hline $60-69$ & 0.082 & 0.097 & 0.073 & 0.042 & 0.049 \\
\hline $70-79$ & 0.107 & 0.126 & 0.058 & 0.069 & 0.054 \\
\hline$\geq 80$ & 0.206 & 0.191 & 0.124 & 0.079 & 0.068 \\
\hline \multicolumn{6}{|l|}{ Distant } \\
\hline $20-29$ & 0.737 & 0.058 & 0.026 & 0.000 & 0.040 \\
\hline $30-39$ & 0.589 & 0.206 & 0.058 & 0.025 & 0.070 \\
\hline $40-49$ & 0.491 & 0.190 & 0.081 & 0.052 & 0.026 \\
\hline $50-59$ & 0.505 & 0.195 & 0.075 & 0.054 & 0.011 \\
\hline $60-69$ & 0.508 & 0.195 & 0.073 & 0.05 & 0.000 \\
\hline $70-79$ & 0.533 & 0.153 & 0.035 & 0.028 & 0.022 \\
\hline$\geq 80$ & 0.674 & 0.089 & 0.068 & 0.096 & 0.002 \\
\hline
\end{tabular}

tory state after treatment. The well history state can lead to an increase in screening rates as individuals diagnosed with CIN are likely to become cautious about CIN and cancer. If cancer progresses, individuals can only move to the cancer cured state if they receive treatment and survive for 5 years. These individuals are assumed to be cancer free and considered to not receive further screening. A death state is present in each status, and all individuals are transferred to the death state 100 years after the start of the cohort.

Because this study aimed to identify strategies that can improve screening uptake, four screening strategies were evaluated: (1) the current strategy, which is the presently followed national cancer screening program in South Korea. In this strategy, all females aged 20 or above receive the Pap smear test biennially, and individuals screened positive obtain further secondary examinations. (2) The strong screening recommendation to target regions strategy, which, in addition to the current strategy, sends strong screening recommendation posts to target populations residing in areas with higher than average mean mortality rates. (3) The regular universal screening recommendation strategy, which dispatches regular screening recommendation posts to all target populations. (4) The strong universal screening recommendation strategy, which mails strong screening recommendations to all target populations. The four strategies are presumed to result in a difference of screening uptake rates: (1) current: biennial Pap to females aged 20 or above (reference), (2) strong screening recommendation by mail to target regions, (effect, 12\% increase in screening uptake; cost, 1,000 KRW per person), (3) regular universal screening recommendation by mail, (effect, $6 \%$ increase in screening uptake; cost, $500 \mathrm{KRW}$ per person), and (4) strong universal screening recommendation by mail (effect, $12 \%$ increase in screening uptake; cost, 1,000 KRW per person).

A cohort of 1,000,000 women aged 20 or above without a history of cervical cancer was included in the simulation model because the national cervical cancer screening program in South Korea targets this group [13]. The model simulated each cohort member based on age-specific cervical cancer incidence and mortality rates. Analysis was conducted using the restricted societal perspective, which accounted for direct medical costs, indirect costs, and caregiving costs, but excluded human productivity costs. 


\section{Model parameters}

Age- and region-specific screening uptake rates were obtained from the 2012 Korean Community Health Survey (KCHS) (Table 1). The KCHS is a nationally representative survey conducted in 253 local districts of South Korea by the Korean Centers for Disease Control and Prevention with the goal of understanding disease prevalence and morbidity patterns. Age- and region-specific mortality rates were calculated by eliminating cancer mortality rates from the general mortality rate reported by Statistics Korea in 2012 (Table 1).

Cervical cancer stages were based on the Surveillance, Epidemiology, and End Results (SEER). As SEER statistics on cervical cancer transition probabilities were unavailable, International Federation of Gynecology and Obstetrics (FIGO) stages were converted to SEER stages using statistics reported in the FIGO annual report [14,15]. Table 2 presents the age- and stage-specific 5-year mortality rates of cervical cancer in South Korea. As SEER cancer stage based transition probabilities were not available in South Korea, this information was obtained by converting FIGO cancer stage based transition probabilities [15].

$\mathrm{HPV}$ infection rates were attained using simulation results reported by Ko et al. [7], who took into consideration rate of HPV infection and sexual experience (Table 3). The CIN transition probabilities were obtained from the National Health Insurance data (Table 3) [16]. Parameters pertaining to the regression probabilities in the precancerous stages were obtained from previous cost effectiveness analysis of cervical cancer in Thailand as no local information was available for Korea (Table 3) $[17,18]$. Cervical cancer transition probabilities were calculated by transforming FIGO stages to SEER stages, as described above (Table 3) [14].

\section{Costs}

Direct and indirect costs were included in this study, as shown in Table $4[19,20]$. Direct medical costs included those incurred from the Pap smear test and, with regard to individuals who screened positive, those associated with colposcopy, biopsy, and HPV DNA tests. The average usage rates of the three examinations were used because information regarding the rate of each examination was not available. Direct tests also included consultation costs and treatment costs for individuals diagnosed with precancerous states or cancer. Treatment costs were calculated based on a previous study conducted by Goldhaber-Fiebert because studies conducted in South Korea did not measure costs by disease stage [21]. Indirect costs included time, transportation, and caregiving costs. Discount rates were assumed to be $1 \%$.
Table 3. Cervical cancer screening program model parameters

\begin{tabular}{|c|c|}
\hline Parameter & Value \\
\hline \multicolumn{2}{|l|}{ HPV infection rate } \\
\hline \multicolumn{2}{|l|}{ Age (yr) } \\
\hline $20-29$ & 0.24 \\
\hline 30-39 & 0.14 \\
\hline $40-49$ & 0.13 \\
\hline $50-59$ & 0.09 \\
\hline $60-69$ & 0.03 \\
\hline$\geq 70$ & 0.01 \\
\hline \multicolumn{2}{|l|}{ Precancerous state transition probability } \\
\hline \multicolumn{2}{|l|}{ State } \\
\hline CIN1 known $\rightarrow$ CIN2/3 known & 8.31 \\
\hline CIN1 unknown $\rightarrow$ CIN2/3 unknown & 13.00 \\
\hline CIN2/3 known $\rightarrow$ Local known & 2.14 \\
\hline CIN2/3 unknown $\rightarrow$ Local unknown & 50.00 \\
\hline \multicolumn{2}{|l|}{ Regression rate } \\
\hline \multicolumn{2}{|l|}{ Regression state } \\
\hline \multicolumn{2}{|l|}{ HPV $\rightarrow$ Well } \\
\hline $20-24 \mathrm{yr}$ & 0.552 \\
\hline $25-29 \mathrm{yr}$ & 0.370 \\
\hline$\geq 30 \mathrm{yr}$ & 0.103 \\
\hline \multicolumn{2}{|l|}{ CIN1 $\rightarrow$ Well } \\
\hline $20-34 \mathrm{yr}$ & 0.1449 \\
\hline$\geq 35 \mathrm{yr}$ & 0.0738 \\
\hline \multicolumn{2}{|l|}{$\mathrm{CIN} 1 \rightarrow \mathrm{HPV}$} \\
\hline $20-34 \mathrm{yr}$ & 0.0161 \\
\hline$\geq 35 \mathrm{yr}$ & 0.0082 \\
\hline CIN2/3 $\rightarrow$ Well & 0.0345 \\
\hline $\mathrm{CIN} 2 / 3 \rightarrow \mathrm{CIN} 1$ & 0.0345 \\
\hline \multicolumn{2}{|l|}{ Cancerous state transition probability } \\
\hline \multicolumn{2}{|l|}{ State } \\
\hline Local unknown $\rightarrow$ Regional unknown & 14.8 \\
\hline Regional unknown $\rightarrow$ Distant unknown & 31.1 \\
\hline
\end{tabular}

\section{Utilities and screening effects}

Age-specific health state utilities were attained using the EuroQol-5D results of the KCHS. Precancerous state patient utilities were adjusted using outcomes from patient surveys [16] and cancer state utilities were calculated based on previous studies $[22,23]$. Sensitivity and specificity of the Pap smear test were determined from a meta-analysis of experimental studies in South Korea [7]. Individuals tested positive on the Pap smear test were assumed to fully participate in secondary tests and the sensitivity of secondary tests were set at $100 \%$. 
Table 4. Cervical cancer screening program cost and utilities parameters

\begin{tabular}{|c|c|}
\hline Cost & Value \\
\hline \multicolumn{2}{|l|}{ Screening $\cos ^{\mathrm{a})}$} \\
\hline \multicolumn{2}{|c|}{ Primary screening costs (total $=48,974)$} \\
\hline Pap smear test & 7,349 \\
\hline Primary diagnosis & 15,159 \\
\hline Consultation & 5,748 \\
\hline Total & 28,256 \\
\hline Transportation (one way) & 6,247 \\
\hline Total $(\times 2)$ & 12,494 \\
\hline Time & 7,954 \\
\hline Postage of results & 270 \\
\hline \multicolumn{2}{|c|}{$\begin{array}{l}\text { Secondary costs due to positive } \\
\text { screening results (total }=89,798 \text { ) }\end{array}$} \\
\hline Re-diagnosis & 11,395 \\
\hline Specialty consultation & 6,267 \\
\hline Total & 17,662 \\
\hline Mean $^{\text {b) }}$ & 32,057 \\
\hline Time $(\times 2)$ & 15,091 \\
\hline Transportation & 6,247 \\
\hline Total & 24,988 \\
\hline \multicolumn{2}{|l|}{ Treatment costs ${ }^{\text {a) }}$} \\
\hline \multicolumn{2}{|l|}{ Precancer treatment } \\
\hline CIN1 treatment & $2,694,607$ \\
\hline CIN2/ 3 treatment & $5,184,292$ \\
\hline \multicolumn{2}{|l|}{ Cervical cancer treatment } \\
\hline Local & $4,585,303$ \\
\hline Regional & $4,907,512$ \\
\hline Distant & $7,860,224$ \\
\hline \multicolumn{2}{|l|}{$\begin{array}{l}\text { Cervical cancer follow up } \\
\text { management at } 1 \mathrm{yr}\end{array}$} \\
\hline Local & $1,642,859$ \\
\hline Regional & $1,641,271$ \\
\hline Distant & $1,650,366$ \\
\hline \multicolumn{2}{|l|}{$\begin{array}{l}\text { Cervical cancer follow up } \\
\text { management at } 2 / 3 / 4 \mathrm{yr}\end{array}$} \\
\hline Local & 871,275 \\
\hline Regional & 870,433 \\
\hline Distant & 875,257 \\
\hline \multicolumn{2}{|l|}{ Utility } \\
\hline \multicolumn{2}{|l|}{ State } \\
\hline Well or well history & 0.763 \\
\hline CIN1 & 0.714 \\
\hline CIN2/3 & 0.711 \\
\hline Local & 0.496 \\
\hline Regional & 0.477 \\
\hline Distant & 0.366 \\
\hline
\end{tabular}

Table 4. Continued

\section{Cost} Value

Sensitivity and specificity of

Pap smear test

CIN1 sensitivity

CIN2/3 sensitivity

0.86

Cancer sensitivity

0.97

Specificity

0.58

$\mathrm{CIN}$, cervical intraepithelial neoplasia. ${ }^{\text {a) }}$ All values are in Korean won (KRW), b)Mean refers to the average colposcopy, biopsy, and human papillomavirus DNA test costs.

\section{Distributional cost effectiveness analysis}

Distributional cost effectiveness analysis aims to adjust the total effectiveness outcome with an inequality index so that strategies with larger health disparity result in lower total effectiveness [24]. A method for distributional cost effectiveness has been introduced by the Centre for Health Economics in 2014, which can be expressed using the Atkinson Inequality Index in Eq. (1) [12]. The Atkinson Inequality Index is a function of inequality aversion, namely public aversion to health disparity, that is measured using public opinion regarding the ideal rate of exchange between the health of individuals with the best and worst health [24]. After calculating the Atkinson Inequality Index for every intervention deliberated in this study, the Atkinson ICER can be measured using Eq. (2), which adjusts the obtained ICER by the Atkinson Inequality Index [24]. The Atkinson ICER can assess which strategy considered in an analysis is the most cost-effective when inequality aversion increases in a society [24]. In this study, the Atkinson ICER was used to account for regional disparities in screening rates and to investigate health inequalities under the cost effectiveness framework.

$$
\begin{aligned}
& A_{\in}=1-\left[\frac{1}{n} \sum_{i=1}^{n}\left(\frac{Q_{i}}{Q}\right)^{1-\in}\right]^{\frac{1}{1-\epsilon}} \\
& A \in \quad \text { Atkinson inequality index for } \in \\
& \text { n Population size } \\
& a_{i} \quad \text { QALY, individual i } \\
& \text { Q OALY, per-capita (mean) } \\
& \in \quad \text { Inequality aversion } \\
& I C E R_{\in}=\frac{C_{2}-C_{1}}{\left(1-A_{\in_{2}}\right) Q_{2}-\left(1-A_{\in 1}\right) Q_{1}} \\
& \text { ICER } \in \quad \text { ICER for } \in \\
& C_{2 / 1} \quad \text { Intervention 2/1's per-capita cost } \\
& A_{\in 2 / 1} \quad \text { Intervention 2/1's Atkinson index } \\
& \mathrm{O}_{2 / 1} \quad \text { Intervention 2/1's per-capita QALY }
\end{aligned}
$$


Table 5. Costs, QALY, and ICER per capita of national cervical cancer screening program strategies

\begin{tabular}{lccccc} 
Variable & Cost & $\begin{array}{c}\text { Incremental } \\
\text { cost }^{\text {a) }}\end{array}$ & Effectiveness & $\begin{array}{c}\text { Incremental } \\
\text { effect }\end{array}$ & ICER \\
Current (baseline) & $25,661,227$ & - & 44.5874 & - & - \\
Strong recommendation to target regions & $26,021,187$ & 359,960 & 44.6363 & 0.0489 & $7,361,145$ \\
Regular universal screening recommendation & $26,169,410$ & 508,183 & 44.6466 & 0.0592 & $8,584,172$ \\
Strong universal screening recommendation & $26,650,816$ & 989,589 & 44.6734 & 0.086 & $11,506,849$ \\
\hline
\end{tabular}

QALY, quality adjusted life years; ICER, incremental cost effectiveness ratio; CIN, cervical intraepithelial neoplasia. ${ }^{\text {a) Costs }}$ are in Korean won (KRW).

\section{Results}

The results of the cost effectiveness analysis of the four compared strategies are presented in Table 5. The strong universal screening recommendation strategy had the highest QALY effectiveness per person, whereas the current strategy incurred the lowest amount of cost. With the threshold set at \$25,990 (KRW 29,901,550), the 2016 GDP of South Korea, the strong universal screening recommendation strategy was the most effective strategy, with an effectiveness of 44.6734 QALY and an ICER value of KRW 11,506,849. In terms of efficiency, the strong screening recommendation to target regions was the most efficient, with an ICER value of KRW 7,361,145.

Table 6 presents the cost effectiveness analysis results of the four strategies by the 16 provincial regions of South Korea. Under the "current" strategy, Seoul had the highest QALYs per capita, whereas Ulsan showed the lowest QALYs per capita. Similar tendencies were found under the strong universal screening recommendation strategy. In the strong screening recommendation to target regions strategy, Busan had the highest QALYs per capita and Ulsan the lowest QALYs per capita.

Fig. 2 presents the results of the distributional cost effectiveness analysis, showing the Atkinson ICER of the four strategies examined. When an identical threshold was utilized, the Atkinson ICER of all four strategies remained under the threshold value as inequality aversion increased. The cost effectiveness of the four strategies also increased as inequality aversion increased, in particular that of the strong screening recommendation to target regions strategy.

\section{Discussion}

Studies have demonstrated that the national cervical cancer screening program in South Korea is cost effective [4-7]. However, screening uptake rates remain low compared to other more economically developed countries and show regional disparities, requiring implementation of effective strategies for improvement. To the best of our knowledge, this is the first study to investigate the required strategies through a cost effectiveness analysis in South Korea. Previous studies have examined investments in educational professionals, local community group education, mailing of pamphlets, and consultation volunteers as approaches to enhance screening rates and found mailings of pamphlets and consultation volunteers to be effective $[10,11,25]$. Considering the requisite resources of the four approaches investigated, mailing of pamphlets was selected as a realistic and applicable scheme. Hence, this study investigated the cost effectiveness of four strategies, current, strong screening recommendation to target regions, regular universal screening recommendation, and strong universal screening recommendation. The results indicated that the ICER values of all four strategies were under the threshold value of $\$ 25,990$ (KRW $29,901,550)$, and that the strong screening recommendation to target regions had the highest efficiency, whereas the strong universal screening recommendation had the highest effectiveness. Therefore, all four strategies investigated rated within the ICER threshold value and may be utilized by policy makers focusing on effectiveness or efficiency.

Another aspect to contemplate by policy makers is the conceivable effect of each strategy on regional disparity because South Korea shows discrepancies between regions in national cervical cancer screening rates. This was reflected using the Atkinson ICER, which indicated the strong screening recommendation to target regions strategy was the most efficient. The ICER value of this strategy decreased the most; thus, its cost effectiveness can be seen to have improved com- 


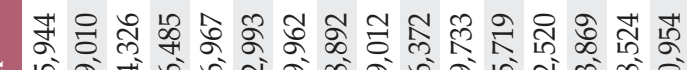

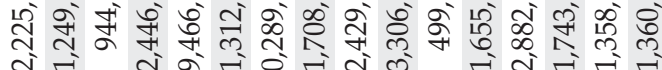
ar $\rightarrow$ त

$\neg-\mathrm{N} r-$

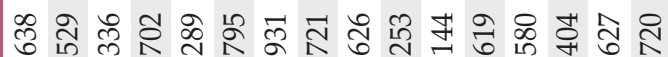

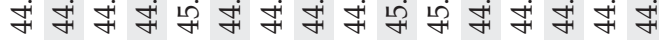

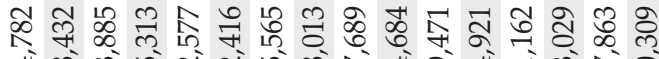
สู่

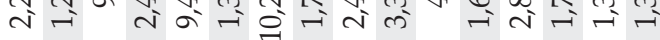

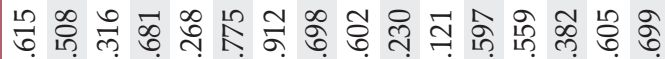

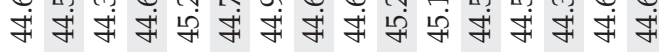

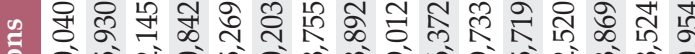

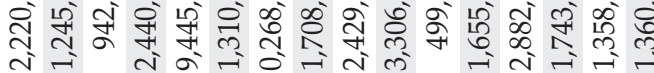

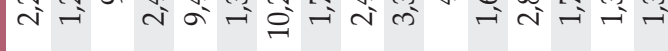

గิ๊

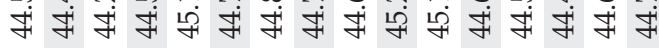

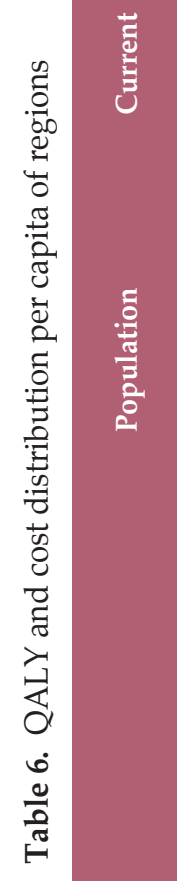

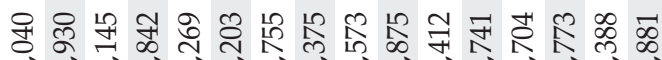

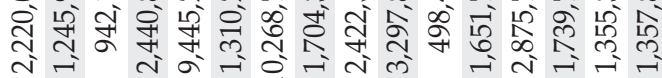

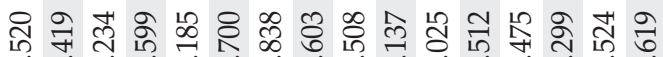

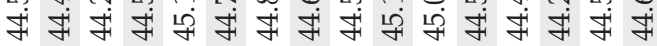

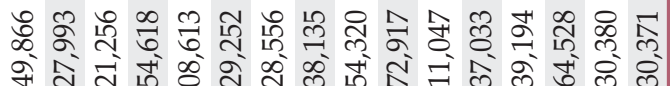

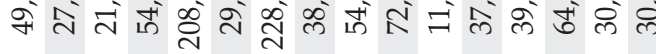




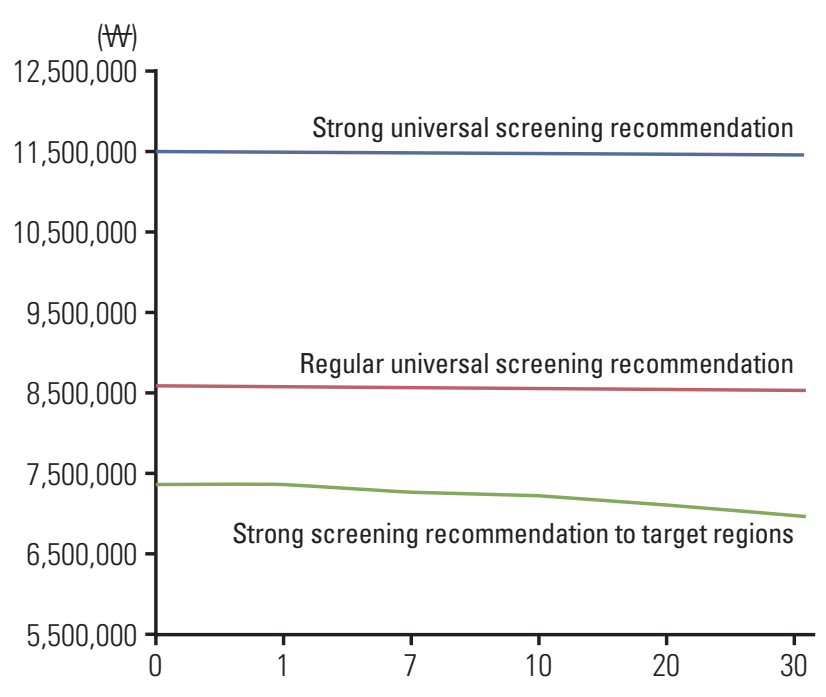

Fig. 2. Atkinson incremental cost effectiveness ratio change on changes in societal inequality aversion.

pared to other strategies. This results as the change in the denominator of the Atkinson ICER $(\Delta \mathrm{E})$ decreases for strategies more unequal to the 'current' strategy and increases for strategies less unequal to the "current" strategy. The tendencies presented in the study findings are similar to those of a previous study that investigated the distributional cost effectiveness of the national colorectal cancer screening program [24]. This study also found the strong screening recommendation to target regions strategy to be most efficient [24]. However, the ICER value decreased in a comparatively steep manner as inequality aversion increased, revealing that health disparities are likely to decline in a comparatively larger amount if the strong screening recommendation to target regions strategy is implemented.

The findings of this study revealed that addressing health inequality aversion can be important in the process of framing health policies. This study offers new insights by investigating the distributional cost effectiveness of the national cervical cancer screening program in South Korea. It is also unique in that it examined different strategies applicable with the goal of improving national cervical cancer screening rates because screening rates in South Korea have been reported to be relatively low, despite its proven cost effectiveness. However, this study also had its limitations. Specifically, age- and region-specific national cervical cancer screening rates were obtained from the KCHS because no other data were available. The KCHS does not distinguish between public and private cervical cancer screening, and screening rates may have been overestimated, which also infers a possible overestimation of the cost measures used in this study. Second, HPV infection, precancerous, and cancer transition probabilities data were not based on South Korean data because they were unavailable. Hence, cost and effectiveness may have again been overestimated as sexual lifestyles tend to be more conservative in South Korea than in other Western countries [16]. Third, although it can be assumed that disease transition probabilities will differ between regions, our study was unable to account for such characteristics because of a lack of data. These assumptions may have led to underestimation of the inequality measured. This limitation has also been mentioned in previous studies and necessitates the measurement of inequality variables. Finally, because this study is the first distributional cost effectiveness study of the national cervical cancer screening program in South Korea, no other results were available to compare with the findings presented.

In conclusion, analysis of the cost effectiveness of the national cervical cancer screening program in South Korea revealed the strong screening recommendation to target regions strategy to be the most cost effective approach. Similar tendencies were found when societal inequality aversion increased under the distributional cost effectiveness analysis, which accounted for regional disparities in screening uptake rates. As efficiency and equity are the two main objectives concurrently sought in healthcare, the findings of this study imply the need to develop appropriate economic evaluation methodologies to assess healthcare policies.

\section{Conflicts of Interest}

Conflict of interest relevant to this article was not reported.

\section{Acknowledgments}

This study was supported by a grant from the National R\&D Program for Cancer Control, Ministry of Health and Welfare, Republic of Korea (No. 1420230). The funding source played no role in the design and conduct of the study; collection, management, analysis, and interpretation of the data; or preparation, review or approval of the manuscript. This study was also supported by the Korea Medical Association Master and Doctor of Philosophy Thesis Support Program. 


\section{References}

1. Statistics Korea. 2014 Mortality statistics results. Daejeon: Statistics Korea; 2015.

2. Luengo-Fernandez R, Leal J, Gray A, Sullivan R. Economic burden of cancer across the European Union: a populationbased cost analysis. Lancet Oncol. 2013;14:1165-74.

3. Tavassoll FA, Devillee P. World Health Organization classification of tumours of pathology and genetics tumours of the breast and female genital organs. Geneva: World Health Organization; 2003. p. 259-90.

4. Jun JK, Choi KS, Jung KW, Lee HY, Gapstur SM, Park EC, et al. Effectiveness of an organized cervical cancer screening program in Korea: results from a cohort study. Int J Cancer. 2009;124:188-93.

5. Shin HR, Park S, Hwang SY, Kim JE, Jung KW, Won YJ, et al. Trends in cervical cancer mortality in Korea 1993-2002: corrected mortality using national death certification data and national cancer incidence data. Int J Cancer. 2008;122:393-7.

6. Cho E, Kang MH, Choi KS, Suh M, Jun JK, Park EC. Costeffectiveness of Korea's National Cervical Cancer Screening Program. Asian Pac J Cancer Prev. 2013;14:4329-34.

7. Ko M, Kim Y, Lee J, Hong S, Sim J, Kim J, et al. Cost-effectiveness of conventional cytology and HPV DNA testing for cervical cancer screening in South Korea. Seoul: National Evidence-based Healthcare Collaborating Agency; 2014.

8. Ministry of Health and Welfare; National Cancer Center. Current state of cancer in statistics. Sejong: Ministry of Health and Welfare; 2015.

9. Cookson R, Drummond M, Weatherly H. Explicit incorporation of equity considerations into economic evaluation of public health interventions: reply to Richardson and Shiell. Health Econ Policy Law. 2009;4(Pt 2):261-3.

10. Huang SL, Chang M. Effects of two teaching programs on workplace women's cervical cancer screening knowledge, health beliefs and behavior. Nurs Res Taipei. 2000;8:111-23.

11. Taylor VM, Hislop TG, Jackson JC, Tu SP, Yasui Y, Schwartz $\mathrm{SM}$, et al. A randomized controlled trial of interventions to promote cervical cancer screening among Chinese women in North America. J Natl Cancer Inst. 2002;94:670-7.

12. Asaria M, Griffin S, Cookson R, Whyte S, Tappenden P. Distributional cost-effectiveness analysis of health care programmes: a methodological case study of the UK Bowel Cancer Screening Programme. Health Econ. 2015;24:742-54.

13. National Health Insurance Service. A guide on national health examination. Seoul: National Health Insurance Service; 2016.

14. Seiffert JE, National Cancer Institute. SEER program: comparative staging guide for cancer. Bethesda, MD: U.S. Department of Health and Human Services, Public Health Service, National Institutes of Health, National Cancer Institute; 1993.

15. Quinn MA, Benedet JL, Odicino F, Maisonneuve P, Beller U, Creasman WT, et al. Carcinoma of the cervix uteri. FIGO 26th Annual Report on the Results of Treatment in Gynecological Cancer. Int J Gynaecol Obstet. 2006;95 Suppl 1:S43-103.

16. Kim Y, An J, Kim Y, Kim J, Park J, Kang S, et al. Cost-effectiveness of human papilloma virus (HPV) vaccines. Seoul: National Evidence-based Healthcare Collaborating Agency; 2012.

17. Myers ER, McCrory DC, Nanda K, Bastian L, Matchar DB. Mathematical model for the natural history of human papillomavirus infection and cervical carcinogenesis. Am J Epidemiol. 2000;151:1158-71.

18. Praditsitthikorn N, Teerawattananon $Y$, Tantivess S, Limwattananon S, Riewpaiboon A, Chichareon S, et al. Economic evaluation of policy options for prevention and control of cervical cancer in Thailand. Pharmacoeconomics. 2011;29:781-806.

19. Health Insurance Review and Assessment Service. National Health Insurance medical care expenses. Wonju: Health Insurance Review and Assessment Service; 2012.

20. Kim Y, Shin S, Park J, Jung Y, Kim J, Lee T, et al. Costing methods in healthcare. Seoul: National Evidence-based Healthcare Collaborating Agency; 2013.

21. Goldhaber-Fiebert JD, Stout NK, Salomon JA, Kuntz KM, Goldie SJ. Cost-effectiveness of cervical cancer screening with human papillomavirus DNA testing and HPV-16,18 vaccination. J Natl Cancer Inst. 2008;100:308-20.

22. Canadian Agency for Drugs and Technologies in Health. Liquid-based techniques for cervical cancer screening: systematic review and cost-effectiveness analysis. Ottawa: Canadian Agency for Drugs and Technologies in Health; 2008.

23. Seol HJ, Ki KD, Lee JM. Epidemiologic characteristics of cervical cancer in Korean women. J Gynecol Oncol. 2014;25:70-4.

24. Lee KS, Park EC. Cost Effectiveness of colorectal cancer screening interventions with their effects on health disparity being considered. Cancer Res Treat. 2016;48:1010-9.

25. Maxwell AE, Bastani R, Vida P, Warda US. Results of a randomized trial to increase breast and cervical cancer screening among Filipino American women. Prev Med. 2003;37:102-9. 